\title{
CMUT Electrode Resistance Design: Modelling and Experimental Verification by a Row-Column Array
}

Havreland, Andreas Spandet; Engholm, Mathias; Tomov, Borislav Gueorguiev; Jensen, Jørgen Arendt; Hansen, Ole; Thomsen, Erik Vilain

Published in:

I E E E Transactions on Ultrasonics, Ferroelectrics and Frequency Control

Link to article, DOI:

10.1109/TUFFC.2019.2906795

Publication date:

2019

Document Version

Peer reviewed version

Link back to DTU Orbit

Citation $(A P A)$ :

Havreland, A. S., Engholm, M., Tomov, B. G., Jensen, J. A., Hansen, O., \& Thomsen, E. V. (2019). CMUT Electrode Resistance Design: Modelling and Experimental Verification by a Row-Column Array. I E E E Transactions on Ultrasonics, Ferroelectrics and Frequency Control, 66(6), 1110-1118 . https://doi.org/10.1109/TUFFC.2019.2906795

\section{General rights}

Copyright and moral rights for the publications made accessible in the public portal are retained by the authors and/or other copyright owners and it is a condition of accessing publications that users recognise and abide by the legal requirements associated with these rights.

- Users may download and print one copy of any publication from the public portal for the purpose of private study or research.

- You may not further distribute the material or use it for any profit-making activity or commercial gain

- You may freely distribute the URL identifying the publication in the public portal 


\title{
CMUT Electrode Resistance Design: Modelling and Experimental Verification by a Row-Column Array
}

\author{
Andreas Spandet Havreland, Mathias Engholm, Borislav Gueorguiev Tomov, Jørgen Arendt Jensen, Ole Hansen \\ and Erik Vilain Thomsen
}

\begin{abstract}
This paper addresses the importance of having control over the resistivity of the electrodes for capacitive micromachined ultrasonic transducers (CMUT) devices. The electrode resistivity can vary depending on the fabrication technology used, and resistivity control becomes especially important in the cases where metal electrodes can not be used. This raises the question: When is the resistivity of an electrode sufficiently low? To answer this question we have developed a simple design criterion. The criterion describes the attenuation of AC signals along a CMUT element. It is shown that the non-dimensional product between angular excitation frequency, resistance, and capacitance $\omega R C$ of an element has to be smaller than 0.35 to ensure an $\mathrm{AC}$ potential drop along the element of less than $1 \%$. The optimal magnitude and directionality of the transmit pressure will be achieved if CMUT elements are designed according to the developed criteria. Hence, the model can be used to estimate device parameters that will ensure the CMUT is suitable for generating ultrasound images. An example is given where the model is used to predict the required electrode thickness for structured electrodes made of Gold, Aluminium, and Indium-Tin-Oxide, respectively. To verify the model, two Row-Column addressed (RCA) CMUT transducers were used to illustrate the effect of high and low electrode resistivity. One transducer had a sufficient electrode resistivity, and the other had an insufficient electrode resistivity. The RCA CMUT transducers were fabricated using fusion bonding, where the top electrode is made of aluminium and the bottom electrode is made of doped silicon. The resistivity of the aluminium top electrode is $2 \times 10^{-6} \Omega \mathrm{cm}$ for both transducers, whereas the resistivity for the bottom electrode is $0.1 \Omega \mathrm{cm}$ for the first transducer and $0.005 \Omega \mathrm{cm}$ for the second transducer. The transducer with low resistivity emits pressure uniformly along both the rows and columns, whereas the transmit pressure field from the other transducer has a uniformly distributed pressure field along the rows, but a decreasing pressure field along the columns due to the high resistivity in the bottom electrode. The pressure drop, along the columns is frequency dependent and has been observed to be $63 \%, 74 \%$, and $82 \%$ for the excitation frequencies $2 \mathrm{MHz}, 4.5 \mathrm{MHz}$, and $7 \mathrm{MHz}$, respectively.
\end{abstract}

This work is financially supported by the Innovation Fund Denmark (705000004B) and by BK Medical (Herlev, Denmark).

A. S. Havreland, M. Engholm and E. V. Thomsen are with the Department of Health Technology, Technical University of Denmark, Kgs. Lyngby, Denmark.

O. Hansen is with the Nanolab Department, Technical University of Denmark, Kgs. Lyngby, Denmark.

B. G. Tomov and J. A. Jensen are with the Center for Fast Ultrasound Imaging, Department of Health Technology, Technical University of Denmark, Kgs. Lyngby, Denmark.

\section{INTRODUCTION}

Capacitive Micromachined Ultrasonic Transducers (CMUT) have shown promising results within the field of ultrasonic medical imaging [1]. Several CMUT papers have been published demonstrating fabrication [2], theoretical models [3], electronics [4], assembly of transducers [1], and safety concerns [5]. The fabrication process of CMUTs can vary significantly from one transducer design to another. However, common for all CMUTs is the overall structure consisting of a substrate, a cavity, and a vibrating plate or membrane. Furthermore, CMUTs have two electrodes, a top and a bottom electrode. The top electrode is typically a metal electrode, whereas the bottom electrode mostly is made of either a metal or doped silicon.

The resistivity of metal electrodes is on the order of $10^{-6} \Omega \mathrm{cm}$ [6], while the resistivity of a silicon electrode varies from $10^{4}$ to $10^{-4} \Omega \mathrm{cm}$ depending on the doping level [7]. The resistance in the electrode has to be sufficiently low to achieve acceptable performance of the CMUTs, which in most cases never becomes an issue for metal electrodes due to their low resistivity. However, in special cases it can become challenging to use even highly doped silicon, particularly for long elements such as elements in a Row-Column Addressed (RCA) array. If the resistivity in an electrode becomes too high, the overall system will behave as a low pass filter, and during operation the applied AC signals will be significantly attenuated along the electrode, leading to variations in the transmit pressure. A non-uniform transmitting element will skew the transmitted field and seriously degrade the receive focusing. Additionally, a larger element resistance decreases the received energy leading to an overall degradation of the SNR in the ultrasound image. Another example where limited electrode resistivity can occur is transparent CMUTs [8], [9]. In such applications the transparent conductor Indium-Tin-Oxide (ITO) is typically used as electrode material, due to its optical transparency and low electrical resistivity. However, the resistivity of ITO is on the order of $10^{-4} \Omega \mathrm{cm}$, hence, two orders of magnitude higher than that of aluminium and gold films, and electrode resistance can therefore become a significant problem in such devices.

To eliminate problems with electrode resistance it would be natural to design a given CMUT device such that the bottom electrode is made of metal. However, this is not always an option, due to process compatibility as high temperature processes do not permit the use of metals. In the literature 

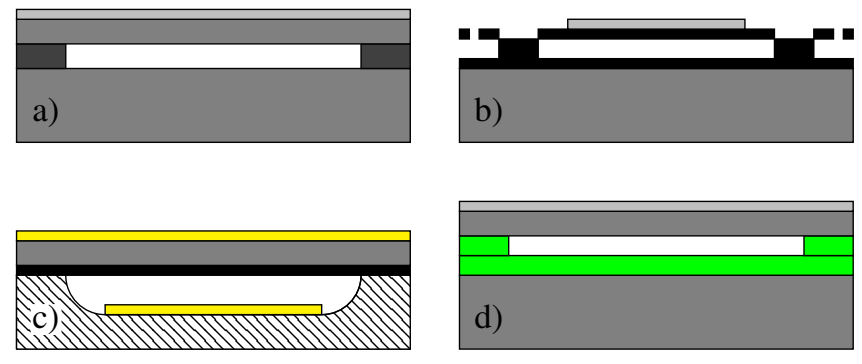

$\mathrm{Si}$

$\mathrm{SiO}_{2}$

$\mathrm{Al}$

$\mathrm{SiN}$

$\mathbb{Q}$ Borosilicate glass

Adhesive

$\square \mathrm{Au}$

Figure 1. Cross sectional view of different CMUT technologies. a) Fusion bonding [10]. b) Sacrificial release [11]. c) Anodic bonding [12]. d) Adhesive polymer bonding [13].

multiple approaches have been reported, and the four most important techniques are fusion/direct bonding [10], sacrificial release [11], anodic bonding [12], and adhesive polymer bonding [13]. Cross sectional views of a CMUT cell fabricated using the four techniques are shown in Fig. 1. A fusion bonded CMUT is sketched in a). This process requires a post annealing temperature on the order of $1000{ }^{\circ} \mathrm{C}$, which excludes the use of a metal bottom electrode. A doped silicon bottom electrode is therefore commonly used instead. A sacrificial release fabricated CMUT is shown in b). This method allows both metal [1] and silicon [11] bottom electrodes. Thus, resistivity problems can be present in this type of CMUTs, if a silicon electrode is used. An anodically bonded CMUT is shown in c), and a gold electrode will be the natural choice for the bottom electrode. Electrode resistance considerations can also be relevant for transparent applications, when ITO is used as electrode material. Finally, an adhesive polymer bonded CMUT is shown in d). This technique is flexible with respect to design, and enables low temperature bonding between all common semiconductor materials. Similar to the sacrificial release an adhesive bonded CMUT can be designed with both a silicon [13], [14] and a metal bottom electrode [15]. This technology has also been used for transparent applications [16], [17], where electrode resistance again can become an issue. In the CMUT applications where problems with electrode resistance can occur, the resistivity and electrode dimensions have to be chosen correctly to obtain full functionality. The minimum required resistivity and electrode dimensions, such as thickness and length, can be estimated by a simple criterion developed in this paper.

The criterion is developed from delay line theory, which is used to predict the attenuation of AC signals along an element, and experimental data, from two RCA transducers, are used to validate the theory. The RCA transducer has two electrodes perpendicular to each other with different resistivities [18]. A RCA transducer is therefore ideal to evaluate the effect of electrode resistance, since the same CMUT cells can be excited by two different electrode configurations. Measuring the AC potential along the bottom electrode is not easy experimentally, however, the effect can be quantified by measuring the transmit pressure along an element. The

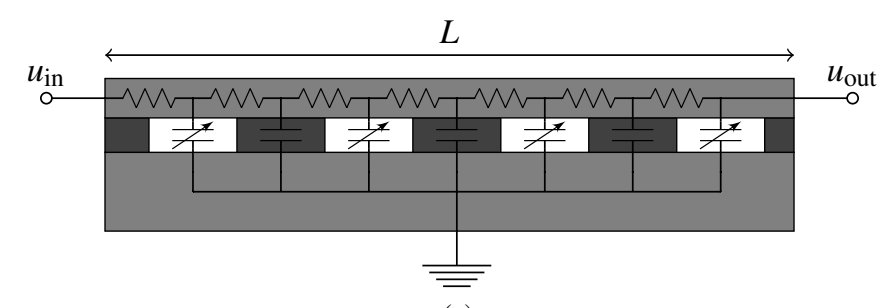

(a)

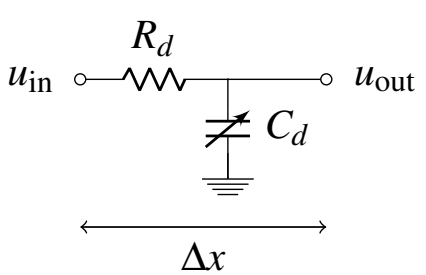

(b)

Figure 2. Model of the Row-Column addressed CMUT array. a) Idealized model of a Row-Column element. The resistance through the element is modelled by resistors, the CMUTs cells are modelled as variable capacitors, and the parasitic capacitance is modelled as constant capacitors. b) When the capacitance of the CMUTs and the parasitic capacitance are merged together, the circuit is described as a Resistor Capacitor (RC) delay line. $R_{d}$ and $C_{d}$ are the resistance and capacitance of a distributed segment of length $\Delta x$ and are related to the total resistance and capacitance by $R_{d}=R / L \cdot \Delta x$ and $C_{d}=C / L \cdot \Delta x$.

model will later be used to estimate the required thickness for different electrode materials. The discussed theory can be used regardless of the fabrication method and applied to both top and bottom electrodes. Publications regarding electrode resistance for CMUTs are limited, and only a brief discussion have been found in [11]. The paper is organized as follows: Section II introduces a delay line theory for CMUT arrays and an explanation of the measurement set-up. Results are discussed and compared to the developed theory in Section III. Finally, Section IV concludes the paper.

\section{METHOD}

\section{A. Modelling}

The potential along CMUT elements can be modelled as a delay line as shown in Fig. 2, where (a) shows a common CMUT design with an equivalent circuits on top. The resistivity of the electrode determines the magnitude of the resistors. The variable capacitors represent CMUTs and the constant capacitors represent the parasitic capacitance. By merging the capacitive components, the electronic circuit can be described by Resistor Capacitor (RC) delay lines as shown in Fig. 2 (b). In this model the resistors and capacitors are assumed to be evenly distributed along the element, $R_{d}$ and $C_{d}$, are resistance and capacitance of a distributed segment of length $\Delta x$. These quantities are related to the total resistance, $R$, and the total capacitance $C$, by

$$
R_{d}=\frac{R}{L} \Delta x \quad C_{d}=\frac{C}{L} \Delta x,
$$

where $L$ is the length of the element. The capacitance of a CMUT element can be mathematically formulated as 


$$
C=C_{p}+n C_{\text {cell }},
$$

where $C_{p}$ is the total parasitic capacitance and $C_{\text {cell }}$ is the capacitance of each of the $n$ CMUT cells. The device capacitance of a CMUT cell can be calculated as described in several papers [11], [19], [20], and is determined by cell geometry and gap height. These parameters are fixed depending on the application. The resistance of an element with an uniform cross-sectional area, $A$, is [7]

$$
R=\frac{\rho L}{A},
$$

where $\rho$ is the resistivity and $L$ is the length of the element. The resistivity in silicon can vary eight orders of magnitude depending on the doping level [7], hence it is a crucial parameter from a design perspective.

In the limit where $\Delta x \rightarrow 0$ and under the assumption of constant capacitance and resistance the voltage distribution $u(x, t)$ in a RC delay line is governed by the diffusion equation [21]

$$
\frac{\partial u(x, t)}{\partial t}=\frac{L^{2}}{R C} \frac{\partial^{2} u(x, t)}{\partial x^{2}},
$$

where $x$ is the position along the length of the element, $t$ is time, and the diffusion coefficient is described by the product $L^{2} / R C$. During operation a CMUT will be subject to the following boundary conditions and initial condition

$$
\begin{aligned}
u(0, t) & =V_{0} g(2 \pi f t) \\
\left.\frac{\partial u(x, t)}{\partial x}\right|_{x=L} & =0 \\
u(x, 0) & =0,
\end{aligned}
$$

where $V_{0}$ is the amplitude of the excitation voltage, and $f$ and $g(2 \pi f t)$ are the excitation frequency and function, respectively. The first boundary condition (5) corresponds to the input signal at the electrode pad. The second boundary condition (6) states that the current density is zero at the end of the electrode, hence, the flux is zero. The initial condition (7) implies zero AC voltage at $t=0$. The diffusion equation does not have a closed form solution for these particular initial and boundary conditions. However, a closed form solution of the transfer function can be found in the frequency domain. After a Fourier transform, $\mathscr{F}$, the diffusion equation (4) can be expressed as:

$$
\frac{\partial^{2} U}{\partial x^{2}}-\frac{\kappa^{2}}{L^{2}} U=0
$$

where

$$
\mathscr{F}\{u\}=U \quad \text { and } \quad \kappa^{2}=i \omega R C .
$$

By imposing the transformed boundary conditions

$$
\begin{aligned}
\mathscr{F}\{u(0, t)\} & =V_{0} G(\omega) \\
\mathscr{F}\left\{\left.\frac{\partial u(x, t)}{\partial x}\right|_{x=L}\right\} & =0
\end{aligned}
$$

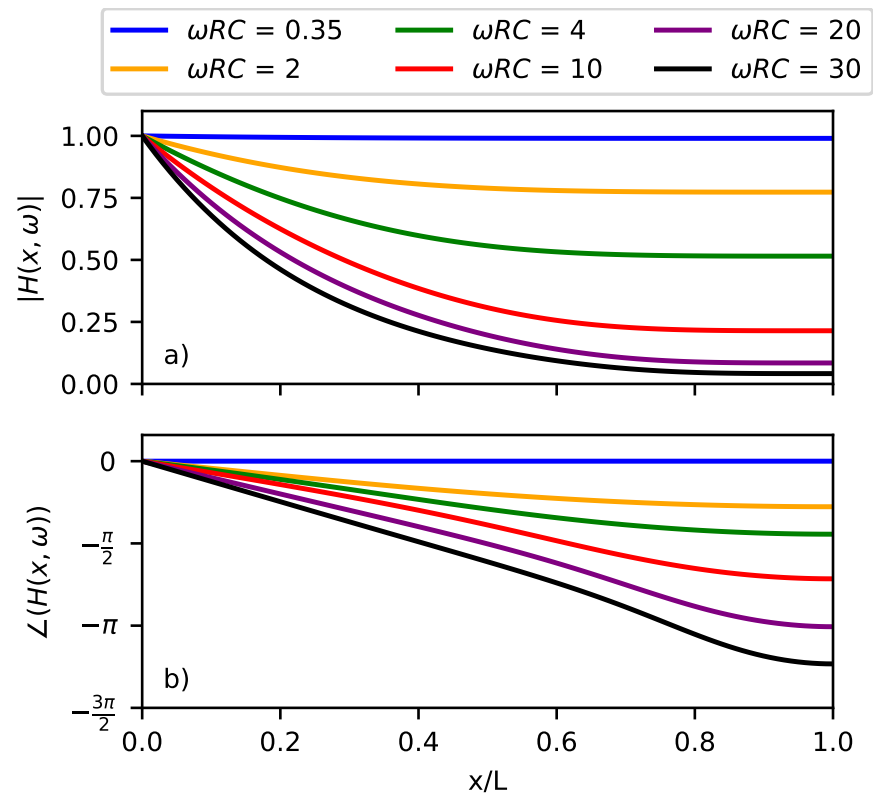

Figure 3. The absolute magnitude and phase of the transfer function as function of normalized distance for six different values of $\omega R C$. a) The magnitude of the transfer function, in the DC case where $\omega R C \rightarrow 0$ the potential becomes uniformly distributed along the element. As $\omega R C$ increases, the $\mathrm{AC}$ potential drop along the element, becomes larger. b) The phase of the transfer function. For $\omega R C \ll 1$ the pressure will approximately be emitted simultaneously along the element, however, for larger values of $\omega R C$ a significant delay is predicted along the element.

the final solution becomes:

$$
U(x, \omega)=V_{0} G(\omega) \underbrace{\frac{\cosh \left(\kappa\left(1-\frac{x}{L}\right)\right)}{\cosh (\kappa)}}_{H(x, \omega)},
$$

where $H(x, \omega)$ is the transfer function of the system.

The product $\omega R C$ is the determining factor for this system, and the product can be understood as an inverse dimensionless diffusion coefficient, see explanation in Appendix A. A visualization of the transfer function is plotted for different values of $\omega R C$ in Fig. 3, for both the absolute magnitude a) and phase b). The absolute magnitude decays along the element, and it translates into a decreasing AC magnitude along the element, hence, the emitted pressure is highest near the contact pad and then attenuated along the element. The attenuating effect becomes more pronounced as $\omega R C$ increases. The phase shift along the element is also influenced by $\omega R C$, so the emission will be time delayed along the element. Equivalently to the magnitude, the higher $\omega R C$ value the more pronounced effect. Consequently, at the end of an element the applied AC signal becomes increasingly attenuated and delayed in time as $\omega R C$ increases. The design criterion is found by evaluating the absolute magnitude of the transfer function at the end of an element, where $H(x, \omega)$ simplifies to

$$
H(L, \omega)=\frac{1}{\cosh (\kappa)} .
$$

A criterion of a $1 \%$ potential drop at the end of an element is used as a guideline to get close to a uniform acoustic pressure 

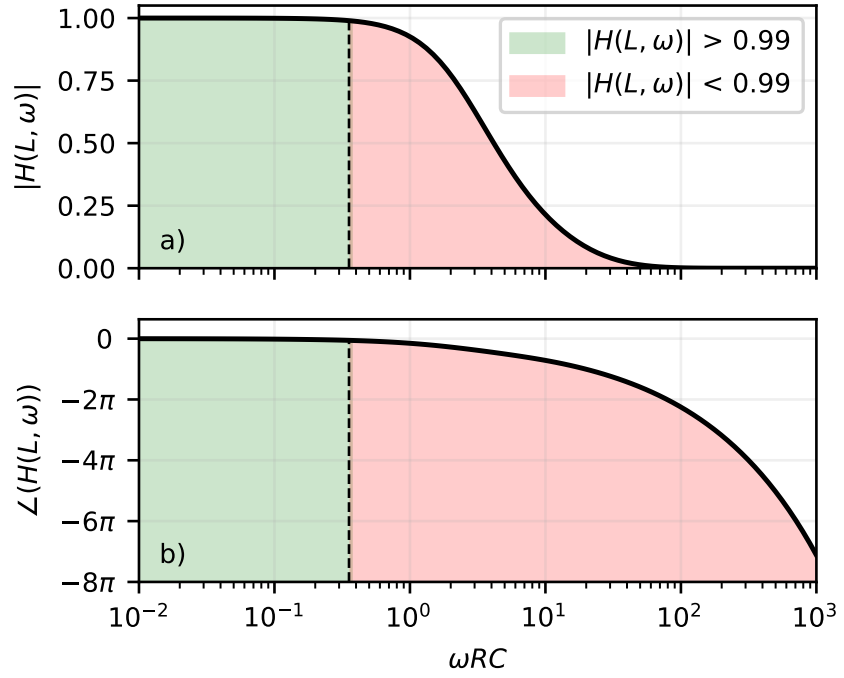

Figure 4. The transfer function at the end of the element $(x=L)$ as function of $\omega R C$. a) The absolute magnitude at the end of an element, where $|H(L, \omega)|=$ 0.99 is indicated with a dashed line. The green region indicates that the magnitude of the transfer function is above above 0.99 and the red region indicates a magnitude less than 0.99 . b) The phase delay at the end of an element. The 0.99 magnitude threshold is also indicated on this plot, and the criterion corresponds to a delay at the end of -0.17 radians or -9.7 degrees.

along the element. In terms of phase shift along the element this criterion corresponds to a delay of -0.17 radians or -9.7 degrees. The magnitude and phase of the transfer function at the end of the element are plotted as a function of $\omega R C$ in Fig. 4 a) and the phase delay is plotted in b). The green area indicates the regime where this criterion is satisfied and red where it is not. A numerical solution of (13) shows that the criterion is met when

$$
\omega R C<0.35 .
$$

CMUTs can be considered as a voltage dependent capacitor, thus, a time dependent voltage signal yields a time dependent capacitance. The time dependence of the capacitance gives rise to non-linearity in this delay line model, and these effects are assumed to be negligible, therefore, all capacitive effects are modelled into a constant capacitor. The capacitance is inversely proportional to the gap height, and during operation of a CMUT the top plate is displaced to either transmit or receive a signal. Hence, changes in capacitance is a necessity for an operational CMUT. However, a constant capacitance assumption is used to obtain a simple analytical closed form model. The largest change in gap size occurs at the resonance frequency, where the constant capacitance assumption is violated most. Away from the resonance the displacement of the top plate decreases, and the validity of the constant capacitance assumption increases. The lower bound of the model where $\omega R C \rightarrow 0$ corresponds to the stationary DC case and is well described by the model. The upper bound where $\omega R C \rightarrow \infty$ will first of all be limited by the underlying assumptions of ideal resistors and capacitors, since inductive components will start to dominate as the frequency increases.
The capacitance and resistance can be measured after the CMUT array has been fabricated. However, estimating $\omega R C$ before fabrication can be cumbersome, especially if the doping profile is varying though the substrate. By introducing the concepts of sheet resistance $R_{\square}$ and capacitance per area $C^{\prime}$, (14) can be rewritten as

$$
\omega R_{\square} C^{\prime} L^{2}<0.35 .
$$

This expression shows a square dependency on the element length, hence, long elements will be influenced significantly by this effect if not designed correctly. The elevation length of 1D arrays is designed by the wavelength multiplied by a factor (typically in the range between 20-30). However, the element length in a RCA array is determined by the number of channels. Thus, by linearly increasing the number of channels the product $\omega R C$ increases with a square dependency. Therefore, problems with resistivity will be much more pronounced in RCA arrays compared to 1D arrays, where these effects rarely will be a problem. Derivation of (15) and an analysis of a varying doping profile can be found in Appendix B.

\section{B. CMUT transducer}

The two transducers used in this work are fully assembled CMUT probes, with identical footprints, and $16.6 \mathrm{~mm}$ long elements. Details of the design, fabrication, and assembly of such probes can be found in the publications [22], [23]. The top electrode is identical for the two probes and made of $400 \mathrm{~nm}$ aluminium with a resistivity on the order of $2 \times 10^{-6} \Omega \mathrm{cm}$. The resistivity for the bottom electrodes differs between the two probes. To distinguish between the two probes, the probe with high resistivity is denoted probe 1 and the probe with lower resistivity is denoted probe 2 . Both probes have a pull-in voltage of $220 \mathrm{~V}$ and $\lambda / 2$ pitch. Probe 1 is a $92+92 \mathrm{RCA}$ array with a center frequency of $4.5 \mathrm{MHz}$ and probe 2 is a $62+62 \mathrm{RCA}$ array with a center frequency of 3.0 MHz. Additional details can be found in Table I. The capacitance is estimated from impedance measurements, and the resistance is calculated using (3) and estimated as the worst-case scenario with respect to the resistivity provided by the SOI-wafer supplier. The difference in capacitance, between the top and bottom electrode, is due to capacitive substrate coupling, as detailed in [24]. The top electrode satisfies in all cases the criterion of $\omega R C<0.35$. However, the bottom electrode has an $\omega R C$ ranging from 9.5 to 33.3 for probe 1 leading to an expected potential drop of $76 \%, 92 \%$, and $96 \%$ for $2 \mathrm{MHz}, 4.5 \mathrm{MHz}$, and $7 \mathrm{MHz}$, respectively.

The bottom electrode for probe 2 has $\omega R C=0.4$, corresponding to an attenuation of $1.2 \%$ along the element. This value does not strictly satisfy the developed criterion, but in an experimental setting, the additional $0.2 \%$ attenuation will not be critical for the overall performance.

\section{Acoustic characterization}

The AC potential drop along the bottom electrode is difficult to measure experimentally due to the encapsulation of CMUTs, so to indirectly verify this effect the transmit pressure field has been measured instead. By using the Row-Column addressing 
Table I

DETAILS ABOUT TOP AND BOTTOM ELECTRODES FOR THE TWO PROBES.

\begin{tabular}{|l|c|c|}
\hline \multicolumn{3}{|c|}{ Probe 1} \\
\hline & Top & Bottom \\
\hline Resistance & $10 \Omega$ & $<6.3 \mathrm{k} \Omega$ \\
\hline Capacitance & $80 \mathrm{pF}$ & $120 \mathrm{pF}$ \\
\hline Resistivity & $3.9 \times 10^{-6} \Omega \mathrm{cm}$ & $<0.1 \Omega \mathrm{cm}$ \\
\hline$\omega R C @ 4.5 \mathrm{MHz}$ & 0.02 & 21.4 \\
\hline \multicolumn{3}{|c|}{ Probe 2} \\
\hline \multicolumn{3}{|c|}{ Top } \\
\hline Resistance & $10 \Omega$ & $<62 \Omega$ \\
\hline Capacitance & $136 \mathrm{pF}$ & $339 \mathrm{pF}$ \\
\hline Resistivity & $3.9 \times 10^{-6} \Omega \mathrm{cm}$ & $<0.005 \Omega \mathrm{cm}$ \\
\hline$\omega R C$ @ $3 \mathrm{MHz}$ & 0.03 & 0.4 \\
\hline
\end{tabular}

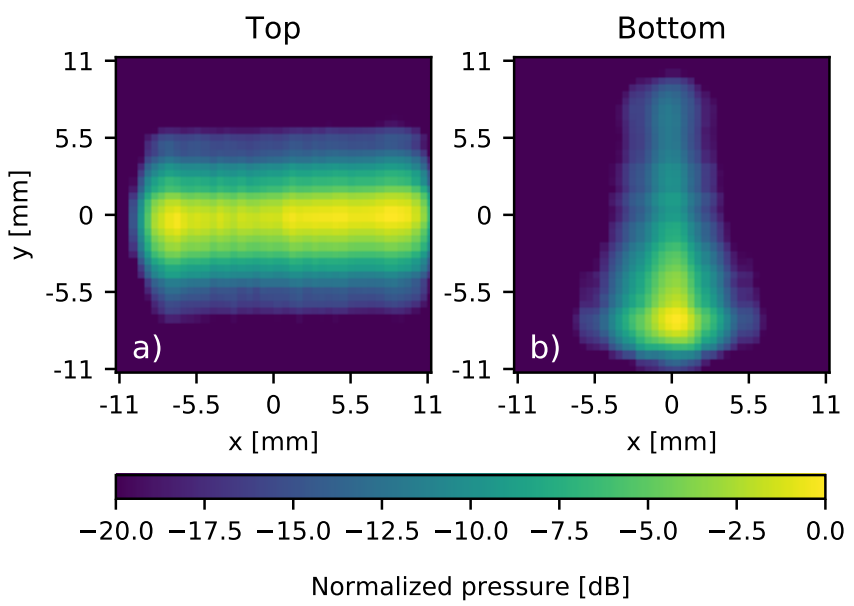

Figure 5. Average peak-to-peak pressure field at 4.5 MHz for probe 1. Pressure field measurement of single top a) and bottom b) electrode. The pressure distribution for the top electrode is uniform along the element. For the bottom electrode the pressure reaches its maximum value near the contact followed by a decaying tendency along the element due to the high resistivity.

scheme, it is possible to actuate the same CMUTs cells, but with two different electrode configurations. The transmit pressure field is measured for all elements individually at a distance of $1 \mathrm{~cm}$ using an AIMS III intensity measurement system (Onda Corp., California, USA) with an Onda HGL0400 Hydrophone connected to the experimental research ultrasound scanner, SARUS [25]. The elements are excited by a 6 cycle sinusoidal pulse at different frequencies. Six cycles were chosen to ensure the correct excitation frequency by minimizing transient effects. The pressure field is mapped by moving the hydrophone in the $x-y$ plane in front of the transducer. The $x-y$ plane is resolved in a $45 \times 45$ grid with a spacing of $0.5 \mathrm{~mm}$. This grid is large enough to capture the entire footprint of the transducer. The pressure fields of all elements (both top and bottom) have been measured by probing all frequencies in each grid point.

\section{RESULTS AND DISCUSSION}

The mean and standard deviation of the peak-to-peak pressure including harmonics have been estimated by spatially crosscorrelating the pressure field from Row element 1 with all other Row elements and likewise for the columns. The averaged peakto-peak pressure field, of single top and bottom electrodes for probe 1, is shown in Fig. 5. The probe is excited at the center

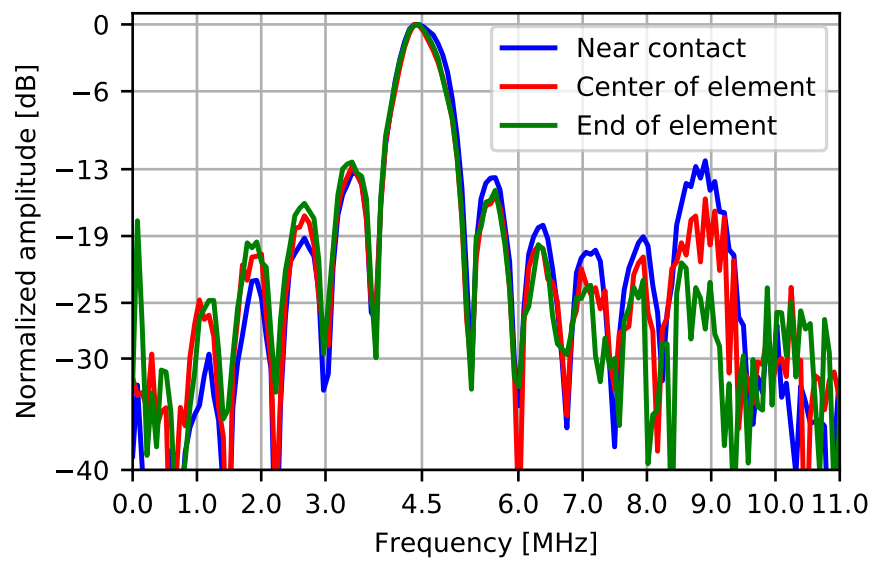

Figure 6. Normalized frequency content for three different positions along an element. The measurement is carried out with probe 1 at $4.5 \mathrm{MHz}$ corresponding to $\omega R C=21.4$. The fundamental to harmonic ratio decreases along the element. Near the contact the second harmonic is suppressed -13 $\mathrm{dB}$, whereas at the center of the element the suppression is $-19 \mathrm{~dB}$, finally, at the end the suppression is observed to be $-25 \mathrm{~dB}$.

frequency of 4.5 MHz and each plot has been normalized to its own maximum and $\mathrm{dB}$ compressed. The top electrodes a) have an $\omega R C$ value of 0.03 satisfying the criterion in (14), and the pressure distribution is observed to be uniformly distributed along the element. The bottom electrodes b) have an $\omega R C$ value of 21.4 and violates the criterion and is expected to attenuate high frequency signals. A significant attenuation is experimentally verified in the pressure distribution, where the maximum pressure is measured at the edge, followed by a decaying tendency along the element. The model predicts an attenuation of $92 \%$ from the electrode pad to the end of the element at $4.5 \mathrm{MHz}$.

To verify that high frequency components are attenuated along the element, the frequency components have been evaluated from three different positions, near the contact, in the center of the element, and at the end of the element. Fig. 6 shows the frequency content normalized to its own maximum for the three positions. The fundamental to harmonic ratio is, as predicted, observed to decreases along the element. The second harmonic is suppressed by $-13 \mathrm{~dB}$ closest to the contact, -19 $\mathrm{dB}$ in the center of the element, and $-25 \mathrm{~dB}$ at the end of the element. Additionally, the low frequency content, relative to the fundamental, is more dominant away from the contact. These measurements verify the low-pass filtering properties of CMUT elements, when $\omega R C$ is not correctly designed.

The pressure drop along an element should be qualitatively comparable with the RC delay line transfer function. The transfer function delay line model is shown for selected values of $\omega R C$ in Fig. 7 a). The mean and standard deviation of the cross-correlated peak-to-peak pressure fields along the center of an element is plotted in Fig. 7 b) and c) for probe 1 and 2, respectively. The solid lines represent the bottom electrodes and the dashed line the top electrodes. Only one pressure profile has been plotted for the top electrodes for probe 1 due to same behavior for the other excitation frequencies. The highest frequency of $7 \mathrm{MHz}$ is used as a representation of the worst-case scenario in terms of $\omega R C$. To 

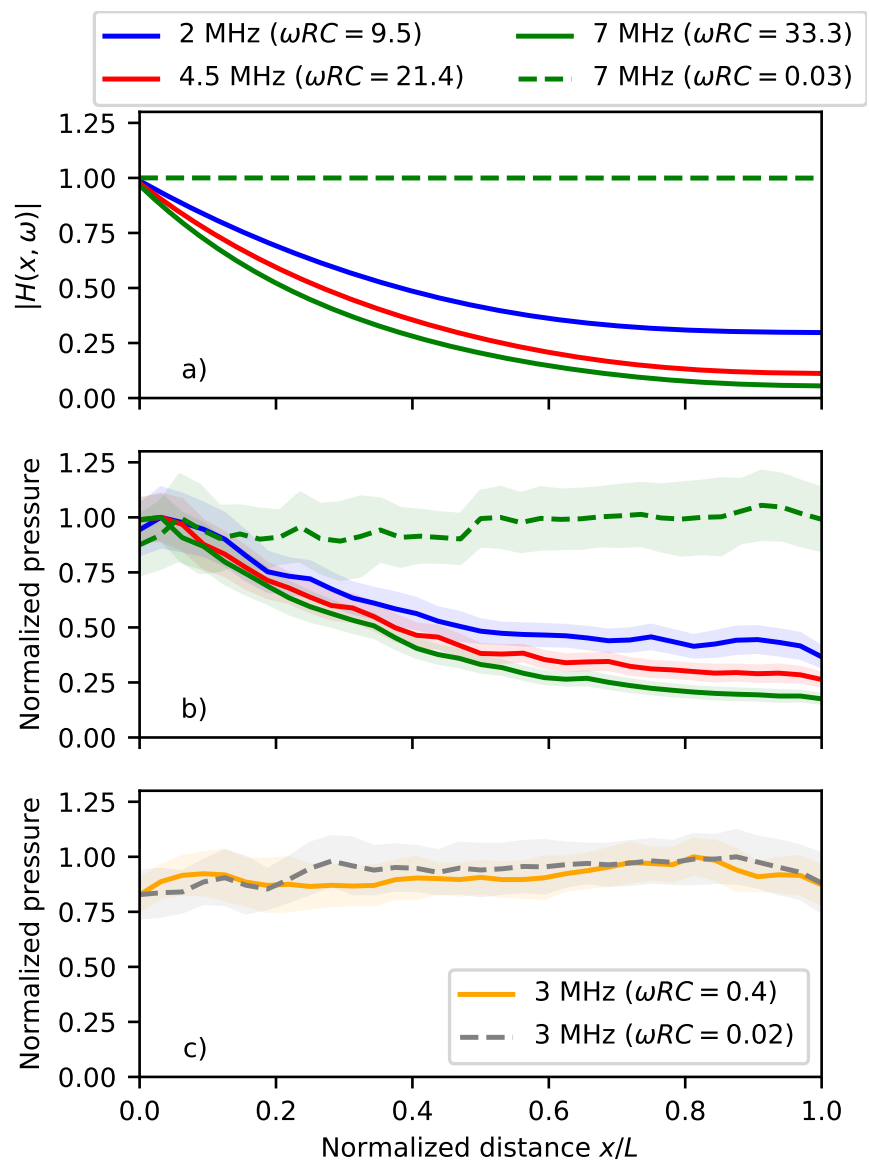

Figure 7. a) Transfer function of the delay line model. b) The measured average peak-to-peak pressure drops along elements for probe 1. c) The measured average peak-to-peak pressure drops along elements for probe 2 . Solid lines indicate the bottom electrode and the dashed lines the top electrode. The shaded area represents one standard deviation. The same qualitative tendency is observed in plot a) and b), which demonstrates consistency between measurement and theory.

obtain a fair comparison between the different frequencies, each pressure profile has been normalized to its own maximum. It is observed that higher values of $\omega R C$ lead to higher reduction of the pressure along the electrode and uniform pressure along the electrode is observed for small value of $\omega R C$, all in good agreement with the transfer function model. The emitted pressure using the bottom electrode from probe 1 is observed to drop $63 \%, 74 \%$, and $82 \%$ for excitation frequencies of $2 \mathrm{MHz}, 4.5 \mathrm{MHz}$, and $7 \mathrm{MHz}$, respectively.

The data acquisition time for the pressure field maps was 4 hours, and long-time-scales-effects are assumed negligible. The standard deviation would vary across the element, if longtime-scales-effects were present. The standard deviation, on Fig. 7 b) and c), is approximately constant along the element for each measurement, thus, the emitted pressure is stable in time.

Attenuation of the applied AC signal along the element will not only affect CMUT elements in transmit. In receive attenuation issues will also occur for pressure signals incident on the end of an element. In addition to attenuation, a higher electrode resistance will also increase the noise floor due to an increased thermal noise voltage, the mean square value

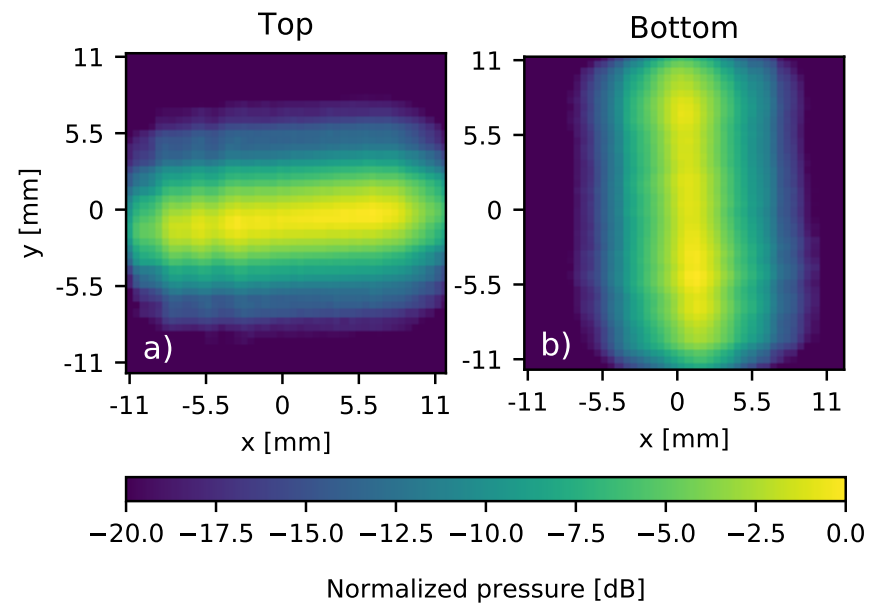

Figure 8. Average peak-to-peak pressure field at $3 \mathrm{MHz}$ for probe 2. Pressure field measurement of single top a) and bottom b) electrode. The pressure field distributions for both top and bottom electrode are uniform along the element, in agreement with an $\omega R C$ of 0.02 and 0.4 for top and bottom, respectively.

of which scales linearly with the resistance. Hence, besides attenuation and delay along the element, a reduction in SNR is also expected, due to the higher resistance.

The electrostatic force for a CMUT scales with $\left(V_{\mathrm{DC}}+V_{\mathrm{AC}}\right)^{2}$, and whenever $V_{\mathrm{AC}} \ll V_{\mathrm{DC}}$ the force will be approximately linear in $V_{\mathrm{AC}}$. This is the reason why the transfer function model maps the normalized pressure quantitatively correct. The discrepancy between Fig. 7 a) and b) could partly be due to an excitation voltage $V_{\mathrm{AC}}$ of $75 \mathrm{~V}$, corresponding to $41 \%$ of the $180 \mathrm{~V}$ applied DC bias, in which case $V_{\mathrm{AC}}^{2}$ becomes non negligible. Non-linear effects could be reduced by lowering the applied AC voltage, however, these parameters are chosen to imitate real operation conditions for medical imaging. Another reason for the discrepancy is the estimate of $\omega R C$, where in particular the resistance is uncertain. The SOI-wafer, used for the bottom electrodes, is specified with an upper bound on the resistivity, and this was used for estimating $\omega R C$. The true $\omega R C$ value could therefore be lower, and thereby decrease the discrepancy between theory and experiment.

To demonstrate the predictability of the model, the same measurements have been carried out on probe 2 . The average pressure field distributions at $3 \mathrm{MHz}$ is shown in Fig. 8 for the top a) and bottom b) electrodes. $\omega R C$ is in this case 0.02 and 0.4 for the top and bottom electrode, respectively. The criterion (14) is nearly satisfied in both cases, and the predicted attenuation is $0.004 \%$ and $1.2 \%$ for top and bottom electrodes, respectively. No significant attenuation is observed for both electrode configurations, which demonstrates that $\omega R C$ can be used as a guideline for the resistance limitation in transducer designs.

Reducing the resistivity will always be beneficial in terms of minimizing $\omega R C$. However, reducing the resistivity in silicon is equivalent to increasing the doping concentration, and as the doping concentration increases, so does the intrinsic stress inside the silicon crystal. In the fabrication process, thermal mismatch after annealing between the layers introduces stress in the CMUT structure. Precise estimates of the stress distribution in the CMUT are difficult to obtain due to a wide temperature 


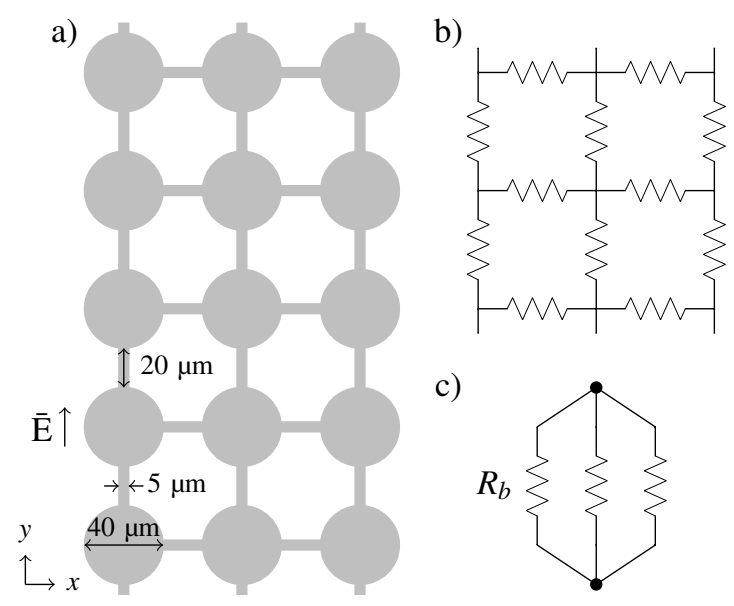

Figure 9. a) Top view sketch of a hypothetical structured electrode to fit the presented design, dimensions for the calculations are included together with assumed direction of the electric field. b) Lumped modelling of the electrode c) Simplified lumped model.

range in the fabrication process. Thus, minimizing of stress prior to the annealing process provides a good prerequisite. CMUT fabrication processes with fusion bonding can benefit from choosing a doping concentration such that $\omega R C$ is in the range between $0.1-0.35$, which will ensure functional CMUTs and at the same time reduced stress in the bottom electrode. The model can be used to calculate the required electrode thickness, and an example for a transducer similar to probe 1 will be given. If the presented CMUT probes, with the same dimensions, had been fabricated using a different method that allowed another bottom electrode, it could be structured and designed as illustrated in Fig. 9 a), where all dimensions are included. The fabrication could in this case be methods based on sacrificial release or anodic bonding. The electrode is composed of circular pads and thin connecting wires, which can be modelled as a resistor network as shown in Fig. 9 b). An estimate of the resistance can be obtained by assuming the electrical field is aligned along the element ( $y$-direction), resulting in zero current flow in the $x$-direction. Hence, all the horizontal resistors can be ignored. This assumption simplifies this circuit to a parallel circuit with three branches, as shown in Fig. 9 c), where a total resistance easily can be calculated. The structured electrode reduces the area of the electrode and thereby its parasitic capacitance. In all estimates of $\omega R C$ the capacitance has been weighted with the reduced electrode area. Each of the vertical resistors in Fig. 9 b) consists of a connecting wire and one disk in series. The resistance of the connecting wires, $R_{\text {wire }}$, is found by (3), and the resistance of a disk can be approximated by (derivation given in Appendix C).

$$
R_{\mathrm{disk}} \approx \frac{\rho}{h} \sqrt{\frac{d+w}{d-w}} \ln \left(\frac{d}{w}\right)
$$

where $\rho$ is the resistivity, $h$ is the film thickness, $d$ is the diameter and $w$ is the width of the connecting wires. A single element has $3 \times 92(=276)$ CMUT cells along the y-direction and three CMUT cells along the $\mathrm{x}$-direction. The resistance in
Table II

RESISTIVITY OF AL, AU, AND ITO TOGETHER WITH THE MINIMUM ELECTRODE REQUIRED THICKNESS.

\begin{tabular}{|l|c|c|}
\hline Material & Resistivity $[\Omega \mathrm{cm}]$ & $h_{\min }[\mathrm{nm}] @ 4.5 \mathrm{MHz}$ \\
\hline Aluminium & $3.9 \times 10^{-6}$ & 58 \\
\hline Gold & $3.5 \times 10^{-6}$ & 50 \\
\hline ITO & $1.8 \times 10^{-4}$ & 2700 \\
\hline
\end{tabular}

each branch, $R_{b}$, in the simplified model is then calculated by

$$
R_{b}=276 \times\left(R_{\text {wire }}+R_{\text {disk }}\right) .
$$

When the resistance of a single branch, $R_{b}$, is known the total resistance can be estimated as a function of electrode thickness and the established criterion (14) can be used to calculate a minimum electrode thickness $h_{\min }$. The resistivity for aluminium, gold, and ITO for $h_{\min } @ 4.5 \mathrm{MHz}$ can be found in Table II. Four point probe measurements have been carried out to determine the resistivity of a $100 \mathrm{~nm}$ aluminium- and gold film, and the ITO resistivity has been found in [26]. Given the design and dimensions in Fig. 9 the estimated minimum electrode thickness for aluminium and gold are $58 \mathrm{~nm}$ and $50 \mathrm{~nm}$, respectively. In the literature the typical thickness of a structured electrode varies from $50 \mathrm{~nm}$ [27] to $200 \mathrm{~nm}$ [28]. Hence, typical metal electrodes will be sufficient for arrays of this size. For transparent CMUT applications the required thickness of ITO is $2.7 \mu \mathrm{m}$, which is impractical in such a fabrication process.

\section{CONCLUSION}

This paper presented a mathematical model to estimate required electrical properties to operate a functional CMUT, and the model has been verified experimentally. Two Row-Column addressed probes were used to demonstrate the effect, one probe with a high resistivity electrodes and another with a low resistivity electrodes. The CMUT probes both had aluminium top electrodes and doped silicon bottom electrodes. The doping level was varied between the two probes corresponding to a bottom electrode resistivity of $0.1 \Omega \mathrm{cm}$ for the first probe and $0.005 \Omega \mathrm{cm}$ for the second probe. High resistivity in the electrode impedes propagation of high frequency AC signals along the element, and the theory of RC delay lines was used to describe the frequency dependent attenuation. High frequency filtering was demonstrated by examining the frequency content at three different positions along an element. For an $\omega R C$ value of 21.4 the fundamental to second harmonic ratio was observed to decrease along the element from $-13 \mathrm{~dB}$ near the contact, to $-18 \mathrm{~dB}$ in the center of the element to $-25 \mathrm{~dB}$ at the end of the element. From this work it can be concluded that a CMUT fabrication process should be designed such that $\omega R C<0.35$ where $\omega$ is the angular excitation frequency, $R$ is the element resistance, and $C$ is the element capacitance. When CMUTs are designed according to this criterion, the amplitude attenuation of the applied AC signal will be reduced less than $1 \%$ along the element. Experimentally this effect was demonstrated for both probes. The low resistivity probe showed a uniform pressure profile along the element, and the high resistivity probe showed a significant decay in the pressure 
profile along the element. For $\omega R C$ equal to $9.5,21.4$, and 33.3 the mean transmit pressure at the end of an element was observed to drop $63 \%, 74 \%$, and $82 \%$, respectively.

\section{ACKNOWLEDGEMENTS}

This work is financially supported by the Innovation Fund Denmark (7050-00004B) and by BK Medical (Herlev, Denmark).

\section{REFERENCES}

[1] A. S. Savoia, G. Caliano, and M. Pappalardo, "A CMUT probe for medical ultrasonography: From microfabrication to system integration," IEEE Trans. Ultrason., Ferroelec., Freq. Contr., vol. 59, no. 6, pp. 11271138, 2012.

[2] M. I. Haller and B. T. Khuri-Yakub, "A surface micromachined electrostatic ultrasonic air transducer," in Proc. IEEE Ultrason. Symp., vol. 2, 1994, pp. 1241-1244.

[3] C. Meynier, F. Teston, and D. Certon, "A multiscale model for array of capacitive micromachined ultrasonic transducers," J. Acoust. Soc. Am., vol. 128 , no. 5 , pp. 2549-2561, 2010.

[4] G. Gurun, P. Hasler, and F. L. Degertekin, "Front-end receiver electronics for high frequency monolithic CMUT-on-CMOS imaging arrays," IEEE Trans. Ultrason., Ferroelec., Freq. Contr., vol. 58, no. 8, pp. 1658-1668, August 2011.

[5] P. Zhang, G. Fitzpatrick, W. Moussa, and R. J. Zemp, "CMUTs with improved electrical safety and minimal dielectric surface charging," in Proc. IEEE Ultrason. Symp., 2010, pp. 1881-1885.

[6] C. Kittel, Introduction to solid state physics. Wiley, 2005.

[7] N. A. Neamen, Semiconductor physics and devices. McGraw-Hill Higher Education, 2003.

[8] X. Zhang, O. J. Adelegan, F. Y. Yamaner, and O. Oralkan, "CMUTs on glass with ITO bottom electrodes for improved transparency," in Proc. IEEE Ultrason. Symp., 2016, pp. 1-4.

[9] X. Zhang, X. Wu, O. J. Adelegan, F. Y. Yamaner, and O. Oralkan, "Backward-mode photoacoustic imaging using illumination through a CMUT with improved transparency," IEEE Trans. Ultrason., Ferroelec. Freq. Contr., vol. 65, pp. 85-94, 2018.

[10] Y. Huang, A. S. Ergun, E. Hæggström, M. H. Badi, and B. T. KhuriYakub, "Fabricating capacitive micromachined ultrasonic transducers with wafer-bonding technology," J. Microelectromech. Syst, vol. 12, no. 2, pp. 128-137, 2003.

[11] A. S. Ergun, Y. Huang, X. Zhuang, Ö. Oralkan, G. G. Yaralioglu, and B. T. Khuri-Yakub, "Capacitive micromachined ultrasonic transducers: Fabrication technology," IEEE Trans. Ultrason., Ferroelec., Freq. Contr. vol. 52, no. 12, pp. 2242-2258, 2005.

[12] F. Y. Yamaner, X. Zhang, and O. Oralkan, "A three-mask process for fabricating vacuum-sealed capacitive micromachined ultrasonic transducers using anodic bonding," IEEE Trans. Ultrason., Ferroelec. Freq. Contr., vol. 62, no. 5, pp. 972-982, 2015.

[13] Z. Li, L. L. P. Wong, A. I. H. Chen, S. Na, J. Sun, and J. T. W. Yeow, "Fabrication of capacitive micromachined ultrasonic transducers based on adhesive wafer bonding technique," J. Micromech. Microeng., vol. 26, no. 11, p. 115019, 2016.

[14] R. Manwar, T. Simpson, A. Bakhtazad, and S. Chowdhury, "Fabrication and characterization of a high frequency and high coupling coefficient CMUT array," Microsyst. Technol., vol. 23, no. 10, pp. 4965-4977, 2017.

[15] A. S. Havreland, M. L. Ommen, C. Silvestre, M. Engholm, J. A. Jensen, and E. V. Thomsen, "BCB polymer based row-column addressed CMUT," in Proc. IEEE Ultrason. Symp., 2017, pp. 1-4.

[16] D. Pang and C. Chang, "Development of a novel transparent flexible capacitive micromachined ultrasonic transducer," Sensors, vol. 17, no. 6 , p. 1443,2017

[17] X. Zhang, O. Adelegan, F. Y. Yamaner, and O. Oralkan, "An optically transparent capacitive micromachined ultrasonic transducer (CMUT) fabricated using SU-8 or BCB adhesive wafer bonding," in 2017 IEEE International Ultrasonics Symposium (IUS), 2017, pp. 1-4.

[18] A. S. Logan, L. L. P. Wong, and J. T. W. Yeow, "2-D CMUT wafer bonded imaging arrays with a row-column addressing scheme," in Proc. IEEE Ultrason. Symp., sep 2009, pp. 984-987.

[19] H. Köymen, A. Atalar, E. Aydogdu, C. Kocabas, H. K. Oguz, S. Olcum, A. Ozgurluk, and A. Ünlügedik, "An improved lumped element nonlinear circuit model for a circular CMUT cell," IEEE Trans. Ultrason. Ferroelec., Freq. Contr., vol. 59, no. 8, pp. 1791-1799, 2012.
[20] M. F. la Cour, T. L. Christiansen, J. A. Jensen, and E. V. Thomsen, "Electrostatic and small-signal analysis of CMUTs with circular and square anisotropic plates," IEEE Trans. Ultrason., Ferroelec., Freq. Contr., p. in press, 2015.

[21] E. Fabricius, Introduction to VLSI design. McGraw-Hill, 1990.

[22] M. Engholm, T. L. Christiansen, C. Beers, J. P. Bagge, L. N. Moesner, H. Bouzari, A. Lei, M. Berkheimer, M. B. Stuart, J. A. Jensen, and E. V. Thomsen, "A hand-held row-column addressed CMUT probe with integrated electronics for volumetric imaging," in Proc. IEEE Ultrason. Symp., 2015, pp. 1-4.

[23] M. Engholm, H. Bouzari, T. L. Christiansen, C. Beers, J. P. Bagge, L. N. Moesner, S. E. Diederichsen, M. B. Stuart, J. A. Jensen, and E. V. Thomsen, "Probe development of CMUT and PZT row-columnaddressed 2-d arrays," Sens. Actuators A: Phys., vol. 273, pp. 121-133, 2018.

[24] M. Engholm, H. Bouzari, J. A. Jensen, and E. V. Thomsen, "Capacitive substrate coupling of row-column-addressed 2-D CMUT arrays," in Proc. IEEE Ultrason. Symp., 2016, pp. 1-4.

[25] J. A. Jensen, H. Holten-Lund, R. T. Nilsson, M. Hansen, U. D. Larsen, R. P. Domsten, B. G. Tomov, M. B. Stuart, S. I. Nikolov, M. J. Pihl, Y. Du, J. H. Rasmussen, and M. F. Rasmussen, "SARUS: A synthetic aperture real-time ultrasound system," IEEE Trans. Ultrason., Ferroelec. Freq. Contr., vol. 60, no. 9, pp. 1838-1852, 2013.

[26] M. Gao, R. Job, D. Xue, and W. R. Fahrner, "Thickness dependence of resistivity and optical reflectance of ITO films," Chin. Phys. Lett., vol. 25 , no. 4 , pp. 1380-1383, 2008 .

[27] S. Olcum, K. Oguz, M. N. Senlik, F. Y. Yamaner, A. Bozkurt, A. Atalar, and H. Koymen, "Wafer bonded capacitive micromachined underwater transducers," in Proc. IEEE Ultrason. Symp., 2009, pp. 976-979.

[28] A. S. Savoia, B. Mauti, G. Caliano, L. Maiolo, A. Minotti, A. Pecora, G. Fortunato, A. Bagolini, and P. Bellutti, "Optimization of the efficiency and reliability of reverse-fabricated CMUT arrays," in Proc. IEEE Ultrason. Symp., 2017, pp. 1-4.

[29] G. S. May and S. M. Sze, Fundamentals of semiconductor fabrication. John Wiley and Sons, Inc., 2004.

\section{APPENDIX A}

The delay line model can be written in a non-dimensional form by introducing the new variables $x^{*}=x / L, t^{*}=\omega t$ and $u^{*}=u / V_{0}$, where the asterisk indicates non-dimensional variables. The non-dimensional version of the differential equation then reads

$$
\frac{\partial u^{*}}{\partial t^{*}}=\frac{1}{\omega R C} \frac{\partial^{2} u^{*}}{\partial x^{* 2}}
$$

where the dimensionless diffusion coefficient is given by

$$
D^{*}=\frac{1}{\omega R C} .
$$

To ensure a highly responsive system, and thereby a more uniform voltage distribution along the element, the diffusion coefficient $D^{*}$ has to be maximized. Notice, this is in full agreement with the parameter found in (12).

\section{APPENDIX B}

$\omega R C$ can be decoupled into lateral and vertical geometry dependence by introducing the concepts of sheet resistance and capacitance per area. The sheet resistance, $R_{\square}$, depends on junction depth $z_{j}$ and the carrier mobility $\mu$ in the following manner [29]

$$
R_{\square}=\frac{1}{q \int_{0}^{z_{j}} \mu(z) N(z) \mathrm{d} z},
$$

where $q$ is the elementary charge, $N$ is the carrier concentration and $z$ is the depth. For SOI wafers, where the doping concentration is constant through the device layer, an analytical 
solution of $R_{\square}$ can be found. In all other cases numerical integration must be used. For an rectangular shaped element the sheet resistance is related to the resistance by

$$
R=\frac{\rho}{h} \frac{L}{W}=R_{\square} \frac{L}{W},
$$

where $W$ is the width of the element. The capacitance per area $C^{\prime}$ is simply the capacitance divided by the cross-sectional area

$$
C=C^{\prime} W L \text {. }
$$

Thus, the product $\omega R C$ can be rewritten by substituting (21) and (22) into (14):

$$
\omega R_{\square} C^{\prime} L^{2}<0.35
$$

$R_{\square}$ and $C^{\prime}$ only have a vertical dependence, whereas all lateral dependencies are now moved into $L^{2} . \omega R_{\square} C^{\prime} L^{2}$ represents a generalized version of $\omega R C$, which can be estimated for all geometries and doping profiles.

\section{APPENDIX C}

The series resistance contribution from the disk shaped element can be calculated using finite elements for any given geometry. An approximate closed form expression, however, is useful when effects of geometrical changes are evaluated. The resistance of the disk shaped element is well approximated by the resistance of two trapezoidal resistance elements in series as illustrated in Fig. 10; each trapezoidal element has a base width equal to the diameter $d$ of the disk, while the top width equals the width $w$ of the connecting wire and the height of the trapezoidal element is $H=\frac{1}{2} \sqrt{d^{2}-w^{2}}=\frac{1}{2} \sqrt{(d-w)(d+w)}$ as seen from elementary geometry.

The cross-section of the conduction path then varies linearly along the element, and the incremental resistance contribution $\mathrm{d} R$ for an element of length $\mathrm{d} y$ becomes

$$
\mathrm{d} R=\frac{\rho}{h} \frac{\mathrm{d} y}{f(y)}=\frac{\rho}{h} \frac{\mathrm{d} y}{w+(d-w) \frac{y}{H}}
$$

where $h$ is the thickness of the conducting material of resistivity $\rho$.

The total resistance of the disk is then approximately

$$
R_{\mathrm{disk}} \approx \frac{2 \rho}{h} \int_{0}^{H} \frac{\mathrm{d} y}{w+(d-w) \frac{y}{H}}=\frac{\rho}{h} \sqrt{\frac{d+w}{d-w}} \ln \left(\frac{d}{w}\right),
$$

where the factor of 2 is due to series connection of two trapezoidal elements.

Compared to Comsol FEM calculations the resistance is overestimated by $\sim 4 \%$ when (24) is used on the geometry of this paper.

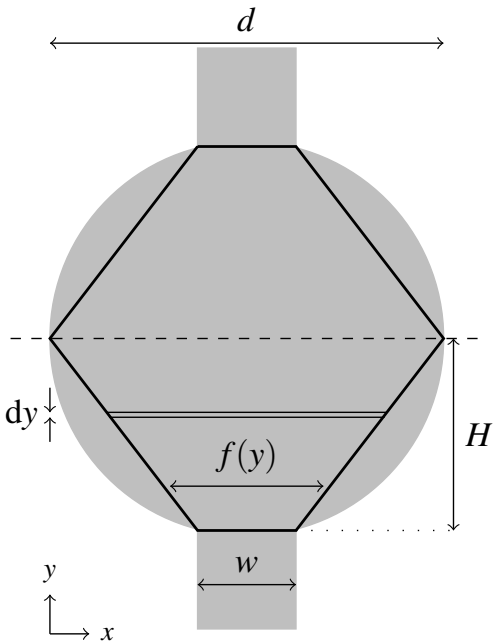

Figure 10. Trapezoidal resistor elements in the disk shaped element. Grey area is the ideal geometry and the solid black lines indicates the approximate disk geometry. 